\title{
Creatine kinase: An enzyme with a central role in cellular energy metabolism
}

\author{
Theo Wallimann ${ }^{\mathrm{a}, *}$, Max Dolder ${ }^{\mathrm{a}}$, Uwe Schlattner ${ }^{\mathrm{a}}$, Michael Eder ${ }^{\mathrm{a}}$, Thorsten Hornemann ${ }^{\mathrm{a}}$, \\ Terry Kraft ${ }^{\mathbf{b}}$, Martin Stolz ${ }^{\text {a }}$ \\ ${ }^{a}$ Institute of Cell Biology, ETH-Hönggerberg, CH-8093 Zürich, Switzerland \\ ${ }^{\mathrm{b}}$ Institute for Molecular Physiology, University Hannover, Hannover, Germany
}

Keywords: Creatine kinase; Cellular energy metabolism; Enzyme

The enzyme creatine kinase (CK), catalyzing the reversible transfer of the $\mathrm{N}$-phosphoryl group from phosphocreatine (PCr) to ADP to regenerate ATP, plays a key role in the energy homeostasis of cells with intermittently high, fluctuating energy requirements, e.g. in skeletal and cardiac muscle, neurons, photoreceptors, spermatozoa and electrocytes. Cytosolic CK isoenzyme(s) (MM-, MB- and BB-CK) are always coexpressed in a tissue-specific fashion together with a mitochondrial isoform. Using biochemical fractionation and in situ localization, one was able to show that the CK isoenzymes, earlier considered to be strictly soluble, are in fact compartmentalized subcellularly and coupled functionally and/or structurally either to sites of energy production (glycolysis and mitochondria) or energy consumption (cellular ATPases, such as the actomyosin ATPase and SR-Ca ${ }^{2+}$-ATPase), thus forming an intricate, highly regulated energy distribution network, the PCr-circuit or PCr-shuttle (Fig. 1, [1]).

This non-equilibrium energy transport model has been challenged, based upon global ${ }^{31} \mathrm{P}-\mathrm{NMR}$ experiments, measuring CK-mediated flux in muscles at different work-loads $[2,3]$. The conclusions reached were that the CK system is in equilibrium with the substrates and behaves like a solution of well-mixed enzymes, that effects of compartmentation were negligible with respect to total cellular bioenergetics and that thermodynamic characteristics of the cytosol could be predicted as if the CK metabolites were freely mixing in solution.

\footnotetext{
* Corresponding author.
}

However, based on the organizational principles of striated muscle, as well as on our findings concerning the highly structured subcellular CK-compartments, this interpretation seemed rather unlikely and thus has been questioned subsequently by a CAVEAT! [4]. In support of this, ${ }^{31} \mathrm{P}-\mathrm{NMR}$ CK-flux measurements with transgenic mice with graded reductions of MM-CK expression in their muscles, show a strikingly unexpected, 'anomalous' CK-flux behaviour [5], indicating that some flux through CK, presumably bound CK, and possibly also some PCr and/or ATP, is NMR-invisible or otherwise not amenable to this analysis $[4,6]$. In the meantime, more evidence from NMR-measurements [7-10], as well as from recent in vivo ${ }^{14}[\mathrm{C}] \mathrm{Cr}$ tracer studies [11], is accumulating in favour of compartmentation of the $\mathrm{CK}$ system and for the existence of different pools of CK substrates. As a matter of fact, it has now become clear that in muscle, $\mathrm{Cr}$ and $\mathrm{PCr}$ molecules do not tumble freely, but display partial orientational ordering, which is in contrast to what is expected for small molecules dissolved in water [7]. Furthermore, ${ }^{31} \mathrm{P}$-NMR saturation transfer experiments with sea-urchin spermatozoa show that the CK-flux increases by a factor of 10-20 upon sperm activation [12]. These specialized sperm cells derive their energy for motility entirely from fatty oxidation within the single large mitochondrion located just behind the sperm head, from where $\mathrm{PCr}$ is diffusing along the 50 $\mu \mathrm{m}$ long sperm tail to fuel the dynein/tubulin ATPase. It is obvious that in these polar, elongated cells, the diffusional limitation of ADP is the key limiting factor 


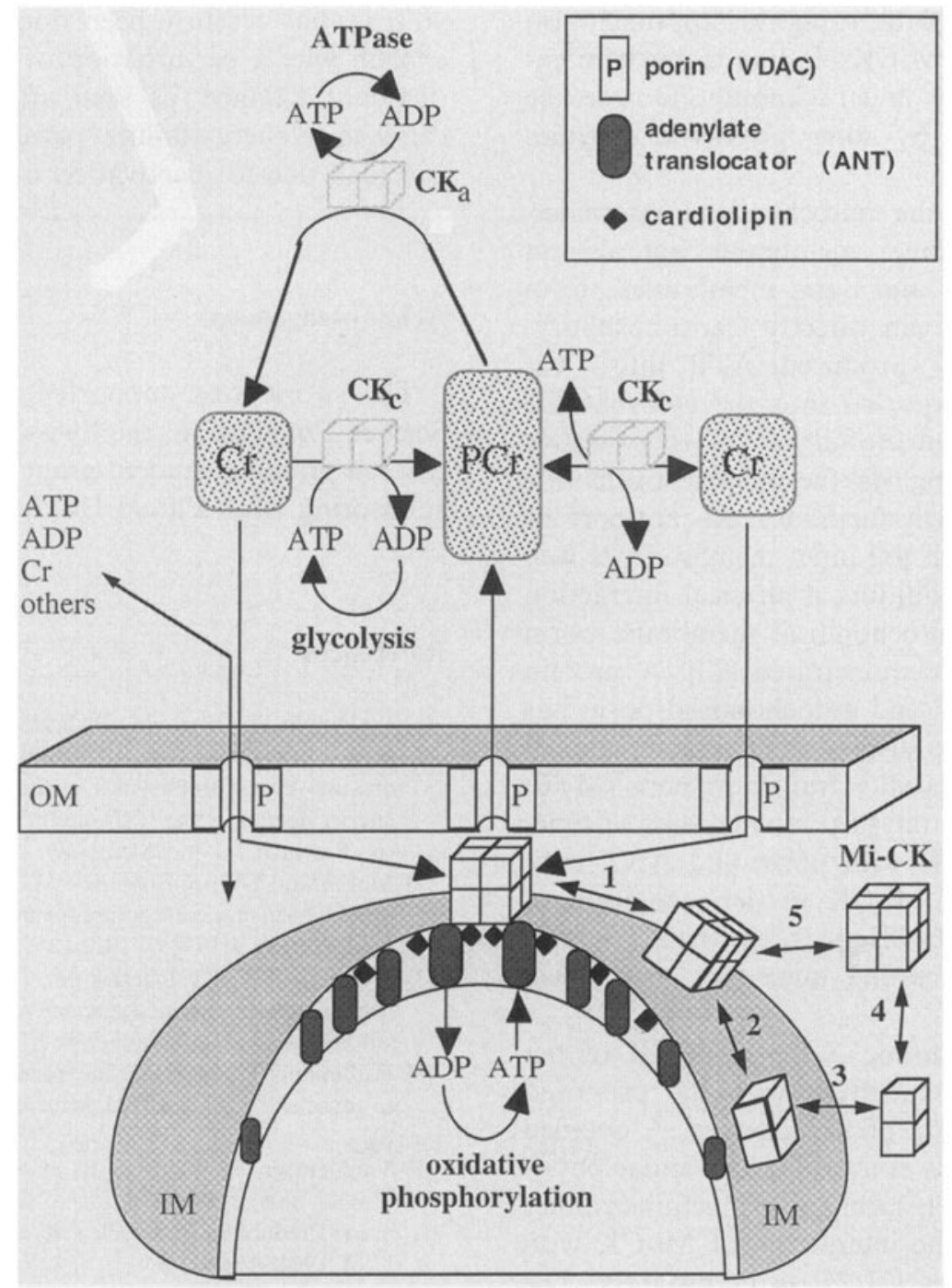

Fig. 1.

with respect to high-energy phosphate provision [13]. In support of the PCr-shuttle model [6,1] the calculated diffusional flux of ADP in these sperm cells are by two and three orders of magnitude smaller than those of ATP and PCr, respectively.

Transgenic CK $(-/-)$ double knock-mice show significantly increased relaxation times of their limb muscles, altered $\mathrm{Ca}^{2+}$-transients in myotubes after stimulation, as well as remarkable remodelling of the contractile apparatus with increased numbers of mitochondria and a grossly over-produced tubular SR membranes [14]. The obvious difficulties of these mice with muscle $\mathrm{Ca}^{2+}$-handling, as the main phenotype, is in line with biochemical and functional data showing that some MM-CK is specifically associated with SR membranes [15], where it is crucial for fueling the energetically highly demanding $\mathrm{Ca}^{2+}$-ATPase $[16,17]$. Therefore, the most crucial function of the CK-system in muscle is related to the energetics of $\mathrm{Ca}^{2+}$. homeostasis.
According to recent findings, AMP-activated protein kinase is able to bind rather tightly to muscle-type MM-CK and phosphorylate the latter to inhibit its activity. AMPK itself is regulated not only by the ATP/AMP ratio, but also by the $\mathrm{PCr} / \mathrm{Cr}$ ratio [18]. This invalidates the long-held dogma that $\mathrm{PCr}$ and $\mathrm{Cr}$ are metabolically completely inert compounds. Thus, AMPK, as an energy sensor system, could represent the missing link for regulation of adaptive metabolic regulation.

Some CK is additionally associated with the myofibril [1]. The isoenzyme-specific association domain of MM-CK with the sarcomeric M-band has been localized by an in situ biochemical approach, using heterologously expressed, fluorescently labelled site-directed mutants, as well as M/B-CK chimaeras for diffusion into chemically skinned muscle fibers [19]. This $\mathrm{M}$-band interaction domain could be narrowed down to two lysine charge-clamps, symmetrically organized on a exposed face of each M-CK monomer 
(unpublished data). New data, using this approach also indicate that the weak MM-CK binding to the sarcomeric $I$-band, observed by in situ immunofluorescence localization is mediated by some glycolytic enzymes [20].

Mi-CK is located in the mitochondrial intermembrane space along the inner membrane, but also at contact sites where inner and outer membranes are in close proximity. Mi-CK can directly transphosphorylate intramitochondrially produced ATP into $\mathrm{PCr}$, which subsequently is exported into the cytosol. The functional coupling of mitochondrial $\mathrm{CK}$ to oxidative phosphorylation, occurring via the adenine nucleotide translocator (ANT), which facilitates the antiport of ATP versus ADP through the inner membrane, is well documented $[21,22]$. In addition, a physical interaction of Mi-CK with outer mitochondrial membrane porin (VDAC) has also been demonstrated [23]. A protein complex containing ANT and mitochondrial porin has recently been described to display the characteristics of the mitochondrial permeability transition pore (MTP) or megachannel [24]. The physical interaction and functional coupling of Mi-CK with porin and ANT indicates an involvement of $\mathrm{Mi}-\mathrm{CK}$ in the regulation of MTP, since octameric Mi-CK in this protein complex [24], plus creatine or creatine analogues, can delay MTP [25].

The recently solved atomic X-ray structure of octameric Mi-CK [26] is consistent with the proposed energy channeling function of this enzyme. A detailed structure/function analysis concerning molecular physiology, catalytic site and mechanism, octamer/dimer equilibrium, as well as the interaction of $\mathrm{Mi}-\mathrm{CK}$ with mitochondrial membranes $[21,27]$ is in progress. The identical top and bottom faces of the octamer contain putative membrane binding motifs likely to be involved in binding of Mi-CK to mitochondrial membranes. The central $20 \AA$ wide channel of the Mi-CK octamer may be of functional significance for the exchange of energy metabolites between mitochondria and cytosol. Furthermore, Mi-CK may follow a 'back door' mechanism by which $\mathrm{PCr}$ is expelled into the central channel of the Mi-CK octamer to facilitate a vectorial transport of $\mathrm{PCr}$ from the mitochondrial matrix into the cytosol. Finally, the $\mathrm{CK} / \mathrm{PCr}$ system is now recognized as an important metabolic regulator during health and disease. Creatine supplementation seems helpful not only for athletes to improve physical performance, but is also emerging as a therapeutic aid for neuromuscular and neurodegenerative diseases. In some of these diseases, especially in mitochondrial myopathies, a compensatory over-expression of $\mathrm{Mi}-\mathrm{CK}$, due to cellular energy deficit, can lead to the formation of pathological intramitochondrial, crystalline Mi-CK inclusions [28]. Furthermore, a pronounced sensitivity of Mi-CK towards reactive oxygen species (ROS), especially perox- ynitrite, has recently been documented [29]. This may explain why a perturbation of cellular pro-oxidant/antioxidant balance, as seen after ischemia/reperfusion, can lead to energy failure, paralleled by chronic calcium overload due to inactivation of CK [29].

\section{Acknowledgements}

This work was supported by the Swiss National Science Foundation, the Swiss Society for Muscle Diseases, a graduate student grant from the ETH-Z and by sponsoring from Careal Holding.

\section{References}

[1] Wallimann T, Wyss M, Brdiczka D, Nicolay K, Eppenberger HM. Intracellular compartmentation, structure and function of creatine kinase isoenzymes in tissues with high and fluctuating energy demands: the 'PCr-circuit' for cellular energy homeostasis. Biochem J 1992;281:21 -40.

[2] McFarland EW, Kushmerick MJ, Moerland T. Activity of creatine kinase in a contracting mammalian muscle of uniform fiber type. Biophy J 1994;67:1912-24.

[3] Wiseman RW, Kushmerick M. Creatine kinase equilibrium follows solution thermodynamics in skeletal muscle: ${ }^{31} \mathrm{P}-\mathrm{NMR}$ studies using creatine analogs. J Biol Chem 1995;270:12428-38.

[4] Wallimann T. ${ }^{31} \mathrm{P}-\mathrm{NMR}$-measured creatine kinase reaction flux in muscle: a CAVEAT!. J Muscle Res Cell Motil 1994;17:17781.

[5] VanDeursen J, Wieringa B, et al. Creatine kinase in skeletal muscle energy metabolism: a study of mouse mutants with graded reduction in muscle CK expression. Proc Natl Acad Sci USA 1994;91:9091-5.

[6] Wallimann T. Dissecting the role of creatine kinase. Curr Biol 1994;1:42-6.

[7] Kreis R, Koster M, Kamber M, Hoppeler H, Boesch C. Peak assignment in localized ${ }^{1} \mathrm{H}$ MR spectra of human muscle based on oral creatine supplementation. Magn Res Med 1997;37:15963.

[8] LeRumeur E, LeTallec N, Kernec F, de Certaines JD. Kinetics of ATP to ADP $\beta$-phosphoryl conversion in contracting skeletal muscle by in vivo ${ }^{31} \mathrm{P}-\mathrm{NMR}$ magnetization transfer. NMR Biomed 1997;10:67-72.

[9] Ntziachristos V, Kreis R, Boesch C, Quistorff B. Dipolar resonance frequency shifts in ${ }^{1} \mathrm{H}$ MR spectra of skeletal muscle: confirmation in rats at $4.7 \mathrm{~T}$ in vivo and observation of changes postmortem. Magn Reson Med 1997;38:33-9.

[10] Williams JP, Headrick JP. Differences in nucleotide compartmentation and energy state in isolated and in sit rat heart: assessment by ${ }^{31} \mathrm{P}-\mathrm{NMR}$ spectroscopy. Biochim Biophys Acta 1996;1276:71-9.

[11] Hochachka PW, Mossey MK. Does muscle creatine phosphokinase have access to the total pool of phosphocreatine plus creatine? Am J Physiol 1998;274:868-72.

[12] VanDorsten F, Wyss M, Wallimann T, Nicolay K. Activation of sea urchin sperm motility is accompanied by an increase in the creatine kinase exchange flux. Biochem $J$ 1997;325:411-6.

[13] Kaldis P, Kamp G, Piendl T, Wallimann T. Functions of creatine kinase isoenzymes in spermatozoa. Adv Develop Biol $1997 ; 5: 275-312$ 
[14] Steeghs K, Wieringa $\mathrm{B}$, et al. Altered $\mathrm{Ca}^{2+}$-response in muscles with combined mitochondrial and cytosolic creatine kinase deficiencies. Cell 1997;89:93-103.

[15] Rossi AM, Eppenberger HM, Volpe P, Cotrufo R, Wallimann T. Muscle type MM-creatine kinase is specifically bound to sarcoplasmic reticulum and can support $\mathrm{Ca}^{2+}$-uptake and regulate local ATP/ADP ratios. J Biol Chem 1990;265:5258-66.

[16] Korge P, Campbell KB. Local ATP regeneration is important for sarcoplasmic reticulum $\mathrm{Ca}^{2+}$-pump function. Am $\mathrm{J}$ Physiol 1994;267:357-66.

[17] Minajeva A, Ventura-Calapier R, Veksler V. $\mathrm{Ca}^{2+}$-uptake by cardiac sarcoplasmic reticulum ATPase in situ strongly depends on bound creatine kinase. Pflügers Arch 1996;432:904-12.

[18] Ponticos M, Lu QL, Morgan JE, Hardie DG, Partridge TA, Carling D. Dual regulation of AMP-activated protein kinase provides a novel mechanism for the control of creatine kinase in skeletal muscle. EMBO J 1998;17:1688-99.

[19] Stolz M, Wallimann T. Myofibrillar interaction of cytosolic creatine kinase (CK) isoenzymes: allocation of $\mathrm{N}$-terminal binding epitope in MM-CK and BB-CK. J Cell Sci 1998;111:120716.

[20] Kraft Th, Nier V, Brenner B, Wallimann T. Binding of creatine kinase to the $I$-band of skinned skeletal muscle fibers is mediated by glycolytic enzymes: an in situ biochemical approach. Biophys J 1996;70:A292.

[21] Schlattner U, Forstner M, Eder M, Stachowiak O, Fritz-Wolf K, and Wallimann T (1998) Functional aspects of the X-ray struc- ture of mitochondrial creatine kinase: a molecular physiology approach. Mol Cell Biochem 184 (in press).

[22] Wyss M, Smeitink J, Wevers R, Wallimann T. Mitochondrial creatine kinase: a key enzyme of aerobic energy metabolism. Biochim Biophys Acta 1992;1102:119-66.

[23] Brdiczka D, Kaldis $P$, Wallimann $T$. In vitro complex formation between the octamer of mitochondrial creatine kinase and porin. J Biol Chem 1994;269:27640-4.

[24] Beutner G, Rück A, Riede B, Welte W, Brdiczka D. Complexes between kinases, mitochondrial porin and adenylate translocator in rat brain resemble the permeability transition pore. FEBS Lett 1997;396:189-95.

[25] O'Gorman E, Beutner G, Dolder M, Koretsky AP, Brdiczka D, Wallimann T. The role of creatine kinase in inhibition of mitochondrial permeability transition. FEBS Lett 1997;414:253-7.

[26] Fritz-Wolf K, Schnyder T, Wallimann T, Kabsch W. Structure of mitochondrial creatine kinase. Nature 1996;381:341-5.

[27] Stachowiak O, Schlattner U, Dolder M, and Wallimann T (1998) Oligomeric state and membrane binding behaviour of creatine kinase isoenzymes: implicaitons for cellular function and mitochondrial structure. Mol Cell Biochem 184 (in press).

[28] O'Gorman E, Fuchs K-H, Tittmann P, Gross H, Wallimann T. Crystalline mitochondrial inclusion bodies isolated from creatine-depleted rat soleus muscle. J Cell Sci 1997;110:1403-11.

[29] Stachowiak O, Dolder M, Wallimann T, Richter Ch. Mitochondrial creatine kinase is a prime target of peroxynitrite-induced modification and inactivation. J Biol Chem 1998;273:16194-699. 